\title{
COMPAÑIA DE PRODUCTOS FRITOS "BOCAOS" LTDA.*
}

\section{Myriam Angulo Olaya}

T a empresa Productos Fritos "Bocaos" Ltda. atraviesa actualmente por una situación coyuntural de estancamiento. El proceso de toma de decisiones estuvo centralizado en la persona de su anterior administrador, quien lijaba los lineamientos y políticas de la empresa y procedía de acuerdo a sus propios intereses o conveniencias, sin tener en cuenta los factores externos o internos que podían afectarla ni las consecuencias negativas de su accionar.

\section{Antecedentes de la organización}

L.a empresa fue fundada en 1967 y se dedica al procesamiento de productos alimenticios, fritos y homeados. denominados pasabocas. Inicialmente salió al mercado con dos productos: papas fritas -en taja-

- Primer premio (companido) en el área de Mercadea. Concurso de casos de CLADEA 1990/ 1991. das y en palillos- y plátano en rodajas. Postcriormente sumó a su oferta las bolas y roscas de queso, los tronquitos, los cubos y los palitos: lucgo, el chicharrón y el cóctel de fritos; después, la bolsa surtida; $y$ finalmente, el mani (Anexo I).

La estructura administrativa inicial era simple. La empresa estaba compuesta por los dueños -pareja de esposos-, sus hijos y tres operarios. La labor de ventas era realizada por el padre-propictario.

Durante muchos años, el desarrollo de la empresa estuvo supeditado al vaivén de su entorno y al juicio de su propictario. quien imponía los productos que la cm. presa debía fabricar basado en su expcriencia anterior como vendedor de alimentos. Lo importante para él era crear nuevos productos, y no le precocupaba cuánto costara fabricarlos. Por lo tanto, todo el dinero obtenido se invertia en los gastos mínimos de la familia y en ensayar nuevos productos o nuevos empaques. 
Durante los cinco primeros años la empresa se manejaba a la deriva, no se ejercía ningún control. Aproximadamente al sexto año, cuando se seguía fabricando sólo la papa frita y el plátano, se entabló contacto con proveedores externos a fin de iniciar la fabricación de las bolas y roscas de queso. Luego se contrató la producción de los cubos bajo las mismas condiciones. Cinco años más tarde se inició la producción del chicharrón, cuyo proceso de fabricación era muy rudimentario e implicaba altos costos cconómicos y humanos. Estando así el negocio, un amigo del propictario, que estuvo en Estados Unidos, le informó sobre la existencia del chicharrón listo para freir. Ambos amigos viajaron a ese país, entraron en contacto con el fabricante y procedicron a importar el producto.

En 1980, las ventas se incrementaron más de loesperado, debido principalmente al esfuerzo de un vendedor "estrella" que por cntonces recién ingresaba a la empresa. Este hecho impulsó al propietario a fabricar las líneas de bolas y roscas de queso, así como los cubos. Se compraron máquinas formadoras de masa, una cilindradora, hornos y dos máquinas $\mathrm{em}$ pacadoras. Además se arrendó una bodega distante tres cuadras de la fábrica.

A partir de entonces, el propietario compraba un vehículo nucvo y creaba una zona nucva cada año. Las ventas continuaron creciendo y en 1983 la empresa adquirió otra máquina empacadora. Dos años más tarde, el propietario decidió invertir en instalaciones para reunir toda la empresa en un único local.

En 1988 nuevamente se adquirí otra máquina cmpacadora, esta vez más grande y sofisticada, $y$ una casa de recreo en
Ibagué, que funciona también como sucursal de la empresa para atender los mercados del lugar. Esta sucursal inició labores con dos vendedores y dos vehículos repartidores, pero a los cinco meses incrementó su fuerza de ventas con cuatro vendedores más y cuatro carros más.

En todo este ticmpo, el gerente-propietario ejercía una dirección vertical, no permitía que se discutiera ninguna de sus órdenes ni que se conociera el destino de los ingresos de la empresa ni los compromisos u obligaciones adquiridos. A su fallecimiento, en junio de 1989, la administración de la empresa quedó en manos de su esposa y de sus nueve hijos, quienes decidieron organizar una junta de socios que fijara las nuevas orientaciones y politicas con las cuales se trabajaría en adelante.

Actualmente la empresa es mancjada por cinco hermanos (cuatro hombres y una mujer), cuatro en Bogotá y uno en la sucursal de Ibagué (ver Anexo 2). Las decisiones están descentralizadas; no existe coordinación entre hermanos, lo que dificulta el normal funcionamiento de la empresa; y no existe visión de lo que los plazos corto, mediano y largo implican para la empresa.

\section{Cuerpo del caso}

La cstructura organizacional y el proceso de toma de decisiones responden a una actitud muy autosuficiente de los propietarios, heredada de su padre y basada en conceptos como éstos: "Yo soy perfecto, nadie sabe lo que yo sé, ni necesito de nadie" Estc fue el dogma con el que el padre-propietario dirigió la empresa; nunca recapacitó en las consecuencias de sus decisiones. Insistía en aplicar sus ideas 
hasta que la misma dinámica del mercado lo convencía de su error.

Actualmente, el proceso de comunicación es continuamente interferido por el poco control que los dirigentes-propietarios ejercen sobre sus emociones. Como por lo general cada uno de ellos hace caso omiso de las decisiones de los demás, las contraordenes son frecuentes. Las continuas discusiones entre los hermanos-propictarios -que no se reprimen ni ante sus trabajadores ni ante cualquier otra persona que se encuentre presente- confunde a los subalternos e interfiere con el normal desarrollo de sus actividades.

Las relaciones interpersonales entre propictarios y trabajadores son malas, como consecuencia del trato prepotente, ofensivo y hasta grosero de los primeros. Cuando alguno de los empleados, de cualquier rango, comete algún error, se le acusa injustamente sin siquiera averiguar las circunstancias en que se produjo el hecho.

Los administradores aún no han tomado conciencia de su responsabilidad de garantizar la estabilidad y el crecimiento de la organización. Distraen su atención en asuntos triviales -discuten si la empresa debe o no comprar una escoba-, aplazando la resolución de lo verdaderamente importante hasta cuando ya no es posible sino tomar una decisión.

Otro aspecto heredado del padre es el arraigado machismo de los propietarios. reflejado en su incapacidad para aceptar sugerencias de su madre y de sus hermanas.

El autoritarismo de cada uno de cllos les impide trabajar en equipo y está causando la paralización de la empresa. Sin embargo, todos están muy ligados afectivamente a ella, prácticamente nacieron y crecieron en su seno, trabajaron hombro a hombro con sus padres desde pequeños. y su mayor deseo es conservarla.

Todos los propietarios, con excepción del jefe de Producción, siguieron carreras universitarias. El gerente general es ingeniero civil; el de Finanzas y Compras, es tecnólogo en Hotelería y Turismo; el administrador de la sucursal de Ibagué estudió siete semestres de Administración de Empresas: $y$ la asistente de Producción, acaba de terminar estudios superiores de Administración de Empresas.

A raíz del fallecimiento del padre, la madre y los hijos perciben una disminu. ción en las ventas; $y$ los costos de producción, financieros y administrativos los agobian cada día más.

\section{Las ventas}

La compania no posee datos históricos sobre el comportamiento de sus ventas; la única información disponible correspon. de al periodo comprendido entre 1986 y 1991. En cuanto al número de artículos producidos, se pasó de $46 \mathrm{en} 1986$ a $36 \mathrm{cn}$ 1991 (ver Anexo 3). Las razones de esta pérdida de diversidad es la dificultad para producir y empacar algunos artículos (los tronquitos) y la escasez de matcria prima para otros (casos de la papa con sabor a salchicha y del mani).

La estructura del departamento de ventas de la cmpresa se muestra en el Anexo 4. La fuerza de ventas cstí organizada en 24 zonas y el mercado que aticnde cs:

- Bogotá y alrededores.

- Ibagué, Huila y alrededores. 
- Villavicencio y alrededores.

- Boyacá y Santanderes.

La compañía clasifica a sus clientes en dos grupos:

a) Clientes de fábrica: son generalmente minoristas de cadena que por los grandes volúmenes que rotan y por su organización centralizada requieren de un tratamiento distinto, pues cllos son quienes fijan sus politicas de pago y las condiciones de manejo y de comercialización de los productos.

Para aceptar un producto, estas empresas estudian las muestras enviadas por el provecdor. Tienen un comitede compras encargado de decidir qué productos deben ser codificados para su venta.

Algunos de estos minoristas de cadena dan mayor espacio para la presentación del producto en los cstantes; por lo tanto, es necesario contar con personal de la empresa para sostener la permanencia del producto. Esta es la labor de las "mercaderistas" y de las "impulsadoras". Sin embargo, los espacios son asignados de acuerdo con la rotación đel producto.

b) Clientes de periferia (clientes tienda a ticnda): son negocios minoristas pequeños atendidos generalmente por su propietario, quien depende integramente de los ingresos que obtiene por los productos vendidos. Generalmente la tienda cstá ubicada en el mismo lugar donde vive el propictario.

El manejo de estos clientes está en función de la empatía existente entre el vendedor y el administrador o propietario. El vendedor debe visitarlos una vez por semana para atender sus pedidos y verifi- car el movimiento de los productos. Las relaciones comerciales se limitan a las acordadas entre el vendedor y el compra. dor. La empresa no otorga crédito; en algunos casos el pago al contado puede extenderse hasta los 30 dias, pero si no se efectúa en ese plazo, la cmpresa descuen. ta el importe del sucldo del vendedor.

Todos los vendedores deben entregar un informe diario de las actividades, visitas y ventas realizadas durante el dia, pero éstos sólo se archivan. La empresa no verifica la autenticidad de la información ni realiza control alguno, tampoco establece planes o programas de ventas. Los vendedores reciben un sucldo equivalente al mínimo legal más 2 por ciento de comisión sobre ventas.

En cuanto a los distribuidores, existen acuerdos verbales de exclusividad: sin embargo, no existe control ni exigencia alguna que garantice el cumplimicnto de las cuotas establecidas. Los vchículos repartidores exhiben anuncios con el nombre de la empresa, pues, por lo general, pertenecieron anteriormente a la empre$\mathrm{sa}$. Sólo los distribuidores que viajan fucra de la ciudad están autorizados para comercializar productos distintos a los de la empresa.

\section{Politica de precios y descuentos}

La empresa otorga descuentos a sus clientes y distribuidores. El descuento establecido para los clientes de fábrica es de 5 por ciento; los clientes de periferia (elientes tienda a tienda) reciben un descuento variable en función del volumen de compra, la frecuencia de los pedidos y el cumplimiento de los pagos, pero el máximo también es de 5 por ciento. A los distribuidores se les otorga un descuento de 20 por ciento. 
Los precios se incrementan una vez al año tomando como referencia el incremento del costo de vida y previo acuerdo entre los principales fabricantes de la industria.

En algunos casos se produce más de un incremento de precios al año, generalmente cuando aumenta brusca $\mathrm{e}$ incontrolablemente el costo de los insumos agropecuarios, muy sensibles a los cambios climáticos y proclives a la escasez.

\section{Proceso de venta}

La empresa vende sus productos a través de dos mecanismos: visitas de los vendedores a los clientes y recibo de pedidos por telćfono.

Cada vendedor debe comunicarse con la empresa por lo menos dos veces al día para averiguar si alguno de sus clientes ha hecho algún pedido, y por la tarde debe acudir a la empresa para llenar el formato "orden de pedido", en el cual especifica las cantidades de cada producto que requiere para cubrir sus ventas del día siguiente.

Este formato es entregado a los auxiliares, quienes colocan los productos solicitados en canastas de plástico. Cada vendedor tiene un número de canastas asignadas $\mathrm{e}$ intercambia las llenas por las vacías. De modo que puede verificarse que el número que sale debe volver a entrar. Estc control tambićn se realiza verificando la orden de pedido y la zona correspondiente.

Cada vez que el vendedor realiza una venta extiende una factura en original y dos copias, una de las cuales entrega al auxiliar de ventas. Este verifica la factura con las órdenes de pedido $\mathrm{e}$ ingresa la información al computador para claborar los resúmenes correspondientes.

\section{Transporte}

La empresa cuenta con una fota de 12 vehículos repartidores, 6 para la ciudad de Bogotá y 6 para su sucursal de Ibagué, además posec un triciclo.

Además, 5 distribuidores utilizan vehículos que anteriormente fueron de propiedad de la empresa. Los otros 5 utilizan triciclos.

El costo de mantenimiento de los vehículos incluye combustible, aceite, cambios de filtro, revisiones periódicas, asco y primas de seguros contra robo y danos a terceros.

\section{Publicidad}

La empresa nunca ha hecho inversión alguna en publicidad. Los únicos medios publicitarios utilizados son los anuncios en los camiones repartidores, los obsequios (agencas y calendarios) de fin de an̂o y las invitaciones a degustaciones que realizan las dos impulsadoras en los almacenes de cadena cuando la gerencia lo considera conveniente.

\section{La industria $y$ la competencia}

La empresa forma parte del sector manufacturero y especificamente de la industria alimenticia. Participa en un mercado de competencia, pues a él concurren varios productores. Dentro del reglón de alimentos, los pasabocas son considerados productos suntuarios y altamente sustituibles; por lo tanto, son muy sensibles a los cambios en los ingresos y en los hábitos de consumo de las personas. Los mayores consumidores de estos productos son individuos de los estratos medio y alto, especialmente ninos y jóvenes -entre 6 y 25 
antos de edad-. Las compras las realizan ellos mismos o sus padres de una manera planificada, pues gran parte de los productos los consumen en el colegio, academia o universidad durante sus horas de descanso.

Las materias primas para la fabricación de estos productos son originarias del sector agropecuario, y por ello no es fácil lograr un abastecimiento adecuado de ellas. En primer lugar, la comercialización de productos agropecuarios está dominada por los intermediarios, quicnes fijan tanto los precios a pagar al agricultor como los precios a cobrar al consumidor. No asumen ningún riesgo y trasladan al precio final los costos por manipulco, transporte y perecibilidad de los productos. $Y$ esta situación sigue siendo así aunque el Estado ha creado instituciones cuyo objetivo es neutralizar la concentración de poder de los intermediarios. En segundo lugar, los cultivos cstán expuestos a una serie de factores incontrolables: sequías, heladas, etc.

La industria de productos fritos enfrenta la competencia de una gran variedad de productos también considerados pasabocas. Se estima que un 70 por ciento de los consumidores compran pasabocas por antojo y en ocasiones especiales o reuniones sociales. La propia industria de productos fritos cuenta con alrededor de 15 fabricantes formalmente cstablecidos y con un número incluso mayor đe informales.

"Bocaos" ocupaba el tercer puesto en cuanto a participación en el mercado, pero ahora ha pasado al cuarto. Actualmente capta el 9 por ciento del mercado en Bogotá, mientras la empresa que ocupa el primer lugar capta el 39 por ciento. La cmpresa realiza el 77 por ciento de sus ventas en Bogotá y el 23 por ciento en zonas fuera de la ciudad. Sus productos líderes son el cóctel de fritos y la bolsa surtida, con los cuales capta el 42 por ciento del mercado específico, es decir, con relación a productos similares de la competencia.

En cuanto a la papa frita cn tajadas (natural y cn sabores), "Bocaos" ocupa el último puesto a pesar que su producto es muy bien aceptado por su sabor. El problema parece tener su origen en la calidad $y$ el empaque.

Con relación al plátano en rodajas, "Bocaos" ocupa el tercer puesto. El primer lugar corresponde al competidor que $\propto c u$. pa el segundo puesto en participación a nivel general, pues el producto viene frito en tajadas y este factor se convierte en una fortaleza para ellos.

El otro producto que goza de gran aceptación entre los consumidores es la línea de cubos, por su sabor y frescura. En esta línea"Bocaos" capta el 40 por ciento del mercado, cmpatando el primer puesto con otro competidor.

Los otros productos fabricados por la empresa, las bolas y roscas de queso y la línea de chicharrón, representan cada uno aproximadamente sólo el 5 por ciento del total de la producción.

La madre-propietaria piensa que sus hijos đebon prestar más atención al árca de ventas. En su opinión, se debe nombrar como jefe de Ventas a una persona con empuje capaz de tomar decisiones trascendentes, pero sabe que sus hijos no lo van a permitir, pues no cuentan con la disposición mental para buscar alternativa a la situación de estancamiento de la cmpresa. 


\section{NOTA PEDAGOGICA}

La calidad de la toma de decisiones y una visión clara de lo que se desea alcanzar a corto, mediano y largo plazo son requisitos fundamentales para la fijación de políticas y pautas de una organización.

Por lo tanto, el presente caso puede ser utilizado en cursos de administración, de mercadeo, de distribución (canales de comercialización y distribución física); en cursos sobre planes y programas para la fuerza de ventas, sobre productos de consumo masivo, sobre desarrollo del producto y segmentación del mercado. También en cursos avanzados que requieran del conocimiento de planes y programas de mercadeo.

Es necesario que quien intervenga $\mathrm{cn}$ el desarrollo del caso posea nociones de mercadco y de investigación de mercados. pues estos conceptos dehen poder ser manejados con claridad.

El caso comienza describiendo $\mathrm{cl}$ origen del problema y luego hace un recuento histórico del desarrollo de la empresa durante sus 24 años de existencia. Expone los factores y situaciones que gencraron su estructura actual, tanto los de indole interna como los de su cntomo.

Se presentan primero los factores que caracterizan la gestión administrativa y lucgo la forma como se manejan los elementos de la mezcla mercadológica (consumidor, producto, precio, distribución y promoción).

El caso finaliza mostrando los aspectos económicos, sociales y políticos del ambiente en que se desenvuelve la empresa.

\section{Objetivo}

Despertar en el estudiante de administración de empresas y particularmente de mercadeo, una capacidad analítica y de investigación que le permita ampliar y profundizar en temas como $\mathrm{el}$ proceso de toma de decisiones en mercadeo, crear nuevos procedimientos o innovar los existentes respecto del mancjo de los elementos de la mezcla mercadológica, y discñar planes estratégicos y programas de mer. cadeo que garantizen la permanencia de una organización.

\section{Preguntas para la discusión}

1. ¿Qué cambios debe realizar ta $\mathrm{cm}$ presa en su departamento de ventas? ¿Cuál sería el diseño más adecuado para permitir su crecimiento?

2. A partir de la información que ofrece el caso, explique cómo podría la empresa realizar una medición del potencial del mercado para seleccionar una adecuada estrategia de mercadeo. ¿Qué información adicional requeriria?

3. ¿Qué objetivo de desarrollo del producto ha estado persiguiendo la empresa desde la introducción de sus productos cóctel de fritus y bolsa surtida?

4. ¿Cree Ud. que hacer una "prucba de concepto" es una ctapa importante para el desarrollo de productos en esta empresa? ¿Qué efectos y reacciones podría ocasionar en la competencia?

5. ¿Sería conveniente que la empresa gastara dinero en cambios de empaque. 
mejoramiento de su proceso productivo y en publicidad? Explique su respuesta.

6. De acuerdo con las características que presenta la aceptación de los productosfabricados por esta empresa, ¿hacia qué deberán orientarse los programas de fijación de precios que se disefien, hacia el incremento de la utilidad o hacia cl mercado?

7. ¿Qué tipos de objetivos de ventas y de distribución serían los más apropiados para esta empresa?

8. A partir de la información del Anexo $2 \mathrm{y}$ de los precios de mercado determine el nivel de ventas en el cual la empresa podrá decidir si es más conveniente utilizar fuerza de ventas o distribuidores.

9. ¿Cuáles đeberán ser los criterios que tenga en cuenta la empresa para determinar la frecuencia apropiada de visitas de venta?

10. Explique cómo podría la empresa evaluar el desempeño de cada vendedor con el actual sistema de control. ¿Qué le haría falta?

\section{Análisis cualitativos y cuantitativos}

- Todos los conocimientos relacionados con la organización empresarial y especificamente con el árca de mercadeo. Los requerimientos, tanto humanos como técnicos, que permitan el desarrollo de una empresa.

- Los conocimientos referentes a crecimiento y participación, tales comonúmero de usuarios, tasa de compra, fidelidad a la marca, comportamiento de compra del consumidor, etc., que permitan a una organización cuantificar el mercado potencial y el suyo propio.

Conocimiento de la distribución de los costos de mercado y de su estructura (variables, fijos: controlables e incontrolables), así como del punto de equilibrio, para poder cvaluar la situación de vendedores vs. distribuidores.

- Conocimientos claros de todos los conceptos que permiten el desarrollo de productos. Conocimientos de investigación de mercados, comportamicnto del consumidor y scgmentación.

- Saber qué es un presupuesto de ventas y cuáles criterios se deben tener en cuenta para la fijación de zonas o territorios, como el número de clientes, los volúmenes de ventas, la accesibilidad a la zona, etc.

- Conocer cómo se estructura un plan de motivación a vendedores: premios, viáticos, capacitación y elementos que ayuden a su buen desempeño.

De la discución se pueden derivar inquietudes con respecto al manejo claro de algunos conceptos, lo que motivaría su revisión. Otros temas serian los conceptos de costos, pronósticos y presupuestos, manejo de conceptos elementales de matemáticas y, sobre todo, el juicio, el sentido común y la creatividad que estimule el caso. 


\section{Anexos COMPAÑIA DE PRODUCTOS FRITOS "BOCAOS" LTDA.}


Anero 1

PRODLCTOS PROCESADOS POR PRODLCTOS FRTTOS "BOCAOS" LTDA.

\begin{tabular}{|c|c|c|c|c|}
\hline Producto & Ingredientes & Presentación & Pes & \\
\hline Línea papa en tajadas & $\begin{array}{l}\text { Sabor natural: } \\
\text { papas seleccionadas. } \\
\text { accite vegetal, sal. }\end{array}$ & $\begin{array}{l}\text { Tamaño popular } \\
\text { Tamanto mediano } \\
\text { Taman̂o familiar } \\
\text { Taman̂o gigante } \\
\text { Taman̂o supergigante } \\
\text { Por libras }\end{array}$ & $\begin{array}{r}20 \\
50 \\
100 \\
280 \\
380 \\
500\end{array}$ & $\begin{array}{l}\mathrm{gr} \\
\mathrm{gr} \\
\mathrm{gr} \\
\mathrm{gr} \\
\mathrm{gr} \\
\mathrm{gr}\end{array}$ \\
\hline & $\begin{array}{l}\text { Sabor picarte: } \\
\text { papas seleceionadas, } \\
\text { aceite vegetal, anti- } \\
\text { oxidantes, sabores } \\
\text { nxturales certifica. } \\
\text { dos. }\end{array}$ & $\begin{array}{l}\text { Tamafio popular } \\
\text { Tamafo familiar }\end{array}$ & $\begin{array}{r}20 \\
100\end{array}$ & $\begin{array}{l}\text { gr } \\
g r\end{array}$ \\
\hline & $\begin{array}{l}\text { Sabor a pollo: } \\
\text { papas seleceionadas, } \\
\text { aceite vegetal, anti- } \\
\text { oxidantes sabores na. } \\
\text { turales cenificados. }\end{array}$ & $\begin{array}{l}\text { Tamaño popular } \\
\text { Tamah̆o familiar }\end{array}$ & $\begin{array}{r}20 \\
100\end{array}$ & $\begin{array}{l}\mathrm{gr} \\
\mathrm{gr}\end{array}$ \\
\hline Linea pap & $\begin{array}{l}\text { Sabor natural: } \\
\text { papa seleccionada, } \\
\text { aceite vegetal y sai. }\end{array}$ & $\begin{array}{l}\text { Tamaho popular } \\
\text { Tamanto familiar } \\
\text { Tamaño gigante } \\
\text { Por libras }\end{array}$ & $\begin{array}{l}20 \\
1 \% 0 \\
200 \\
500\end{array}$ & $\begin{array}{l}\mathrm{gr} \\
\mathrm{gr} \\
\mathrm{gr} \\
\mathrm{gr}\end{array}$ \\
\hline Línea plátano en rodajas & $\begin{array}{l}\text { Plátano selecciona- } \\
\text { do, aceite vegetal y } \\
\text { sal. }\end{array}$ & $\begin{array}{l}\text { Tamaño mediano } \\
\text { Tamaño especial } \\
\text { Tamahto familiar } \\
\text { Por libras }\end{array}$ & $\begin{array}{r}30 \\
60 \\
140 \\
500\end{array}$ & $\begin{array}{l}\mathrm{gr} \\
\mathrm{gr} \\
\mathrm{gr} \\
\mathrm{gr}\end{array}$ \\
\hline $\begin{array}{l}\text { Línea chicharnón } \\
\text { (trode cuero de cerdo) }\end{array}$ & $\begin{array}{l}\text { Cucro de cerdo, } \\
\text { aceite vegetal y sal. }\end{array}$ & $\begin{array}{l}\text { Tamsîo popular } \\
\text { Tamafio mediano } \\
\text { Tamaho familiar }\end{array}$ & $\begin{array}{l}10 \\
40 \\
65\end{array}$ & $\begin{array}{l}g r \\
g r \\
g r\end{array}$ \\
\hline Linca cóctel de fritos & $\begin{array}{l}\text { Plstano, papa, cuero } \\
\text { de cerdo selecciona. } \\
\text { do, aceite vegetal y } \\
\text { sal. }\end{array}$ & $\begin{array}{l}\text { Tamaho familiar } \\
\text { Tamaito supergigante }\end{array}$ & $\begin{array}{l}160 \\
480\end{array}$ & $\begin{array}{l}g r \\
g r\end{array}$ \\
\hline 1.ínca bolas o & $\begin{array}{l}\text { Almidon de yuca, } \\
\text { queso, huevos, leva- } \\
\text { dura. }\end{array}$ & $\begin{array}{l}\text { Tamano populat } \\
\text { Tamaho familiar } \\
\text { Tamaho gigantc }\end{array}$ & $\begin{array}{l}10 \\
35 \\
80\end{array}$ & $\begin{array}{l}g r \\
g r \\
g r\end{array}$ \\
\hline Línca rọscas de queso & $\begin{array}{l}\text { Almidón de yuca, } \\
\text { queso, hucvos y le- } \\
\text { vadura. }\end{array}$ & $\begin{array}{l}\text { Tamafio populat } \\
\text { Tamaño mediano } \\
\text { Tamaño gigante }\end{array}$ & $\begin{array}{l}10 \\
35 \\
60\end{array}$ & $\begin{array}{l}g r \\
g r \\
g r\end{array}$ \\
\hline Línea cubos (cojincitos) & $\begin{array}{l}\text { Ilarina de trigo, } \\
\text { accite vegetal, leva. } \\
\text { dura, margarina, sal } \\
\text { pưmienta, azúcar. }\end{array}$ & $\begin{array}{l}\text { Tamaijo popular } \\
\text { Tamaño mediano }\end{array}$ & $\begin{array}{l}30 \\
45\end{array}$ & $\begin{array}{l}g r \\
g r\end{array}$ \\
\hline Línea bolsa surtida & $\begin{array}{l}\text { Combinación de } 10 \\
\text { paquetes de pasabo- } \\
\text { cas: papa natural, } \\
\text { picanse, pollo, plä- } \\
\text { tano, cojincitos de } \\
\text { harina de trigo, bo- } \\
\text { las y roscas de que- } \\
\text { so, }\end{array}$ & & & \\
\hline
\end{tabular}



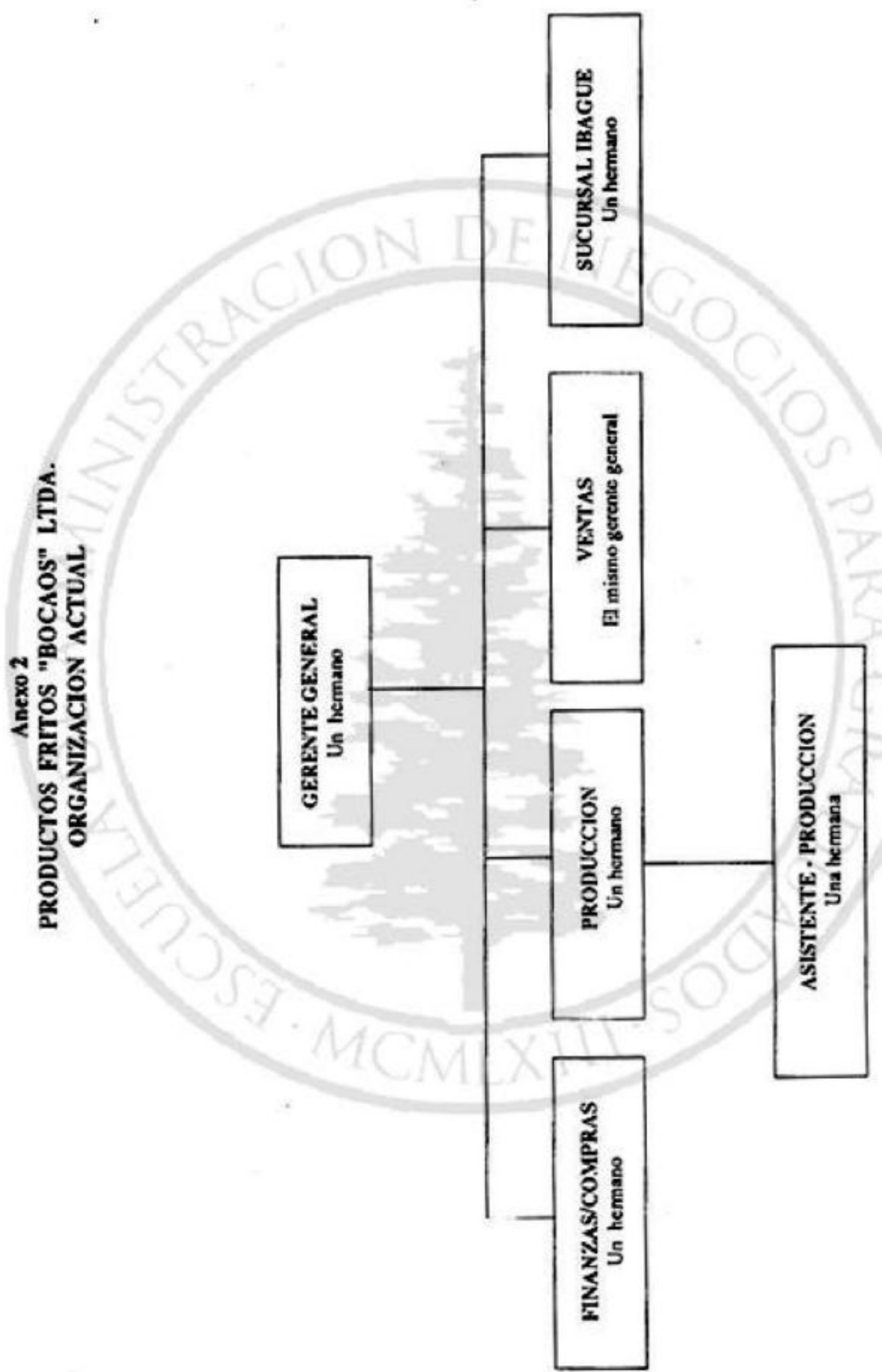


\section{Anexo 3 \\ CLADRO COMPARATIVO ENTRE VENTAS 1986 Y VENTAS 1990 \\ UNTDADES MENSUALES PROMEDIO}

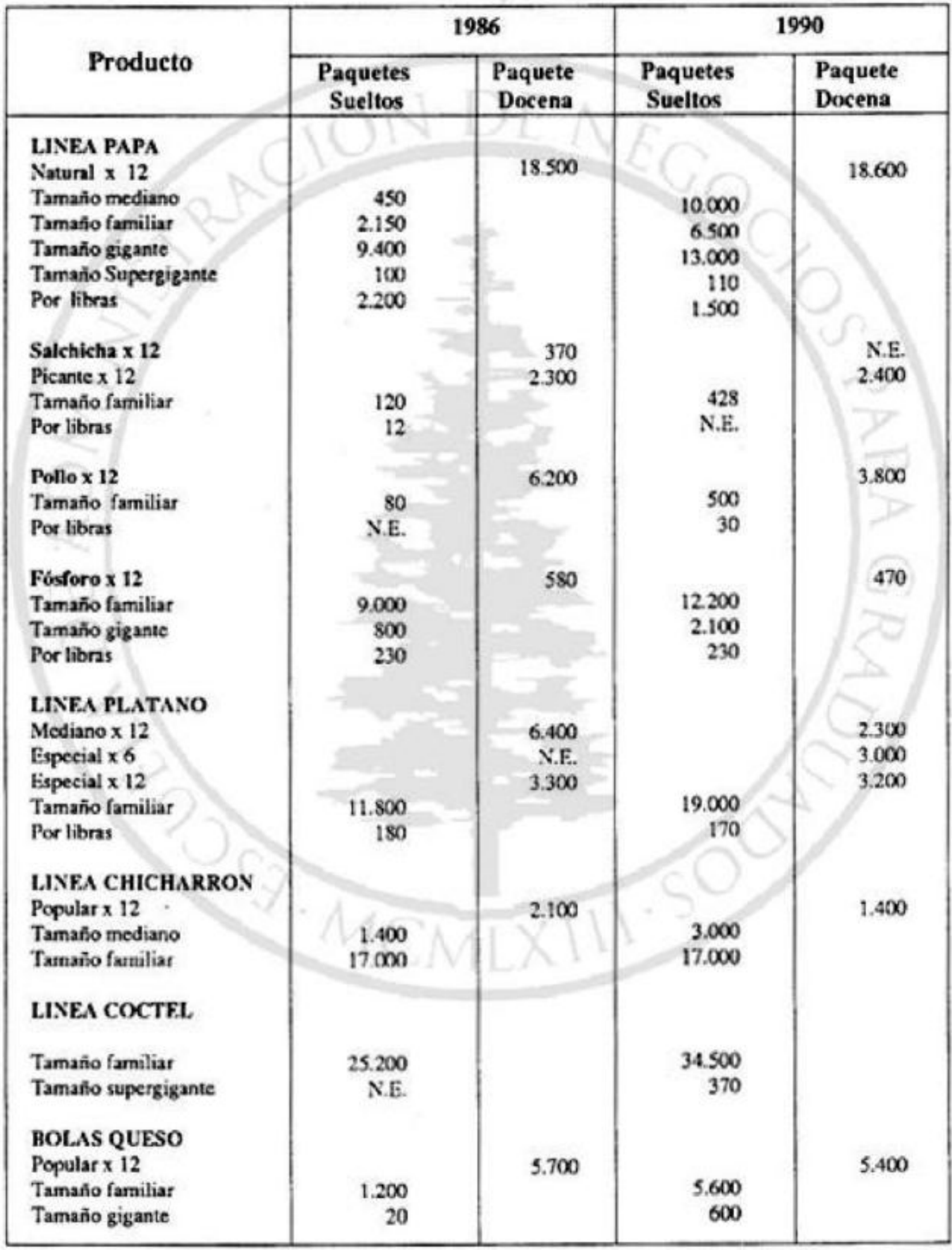

Continuia.. 
Continuación Anexo 3

\begin{tabular}{|c|c|c|c|c|}
\hline \multirow{2}{*}{ Producto } & \multicolumn{2}{|c|}{1986} & \multicolumn{2}{|c|}{1990} \\
\hline & $\begin{array}{l}\text { Paquetes } \\
\text { sueltos }\end{array}$ & $\begin{array}{l}\text { Paquete } \\
\text { docena }\end{array}$ & $\begin{array}{l}\text { Paquete } \\
\text { sueltos }\end{array}$ & $\begin{array}{c}\text { Paquete } \\
\text { docena }\end{array}$ \\
\hline $\begin{array}{l}\text { ROSCAS QUESO } \\
\text { popular } \times 12 \\
\text { Tamaño familiar } \\
\text { Tamaño gigante } \\
\text { TRONQUTrOS } \\
\text { Modiano } \times 6 \\
\text { Modiano } \times 12 \\
\text { Tamafio familiar } \\
\text { LINEA CUBOS } \\
\text { Popular } \times 12 \\
\text { Mediano } \times 6 \\
\text { Mediano } \times 12 \\
\text { Tamaño familiar } \\
\text { Palito familiar } \\
\text { LINEA MANI } \\
\text { Dulce } \times 12 \\
\text { Dulce faniliar } \\
\text { Salado } \times 12 \\
\text { Salado familiar } \\
\text { BOLSA SURTIDA }\end{array}$ & $\begin{array}{r}30 \\
300 \\
20 \\
1.700 \\
18.700\end{array}$ & $\begin{array}{r}3.100 \\
300 \\
\\
\text { N.E. } \\
19.000 \\
16.200 \\
\\
370 \\
900\end{array}$ & $\begin{array}{l}\text { N.E. } \\
\text { N.E. } \\
25.500\end{array}$ & $\begin{array}{r}\text { N.E. } \\
\text { N.E. } \\
\\
240 \\
18.600 \\
12.000\end{array}$ \\
\hline
\end{tabular}

N.E. = No existe. Se trata de productos que en 1986 aún no producia la empresa o de productos que en 1990 ya no se están produciendo. 


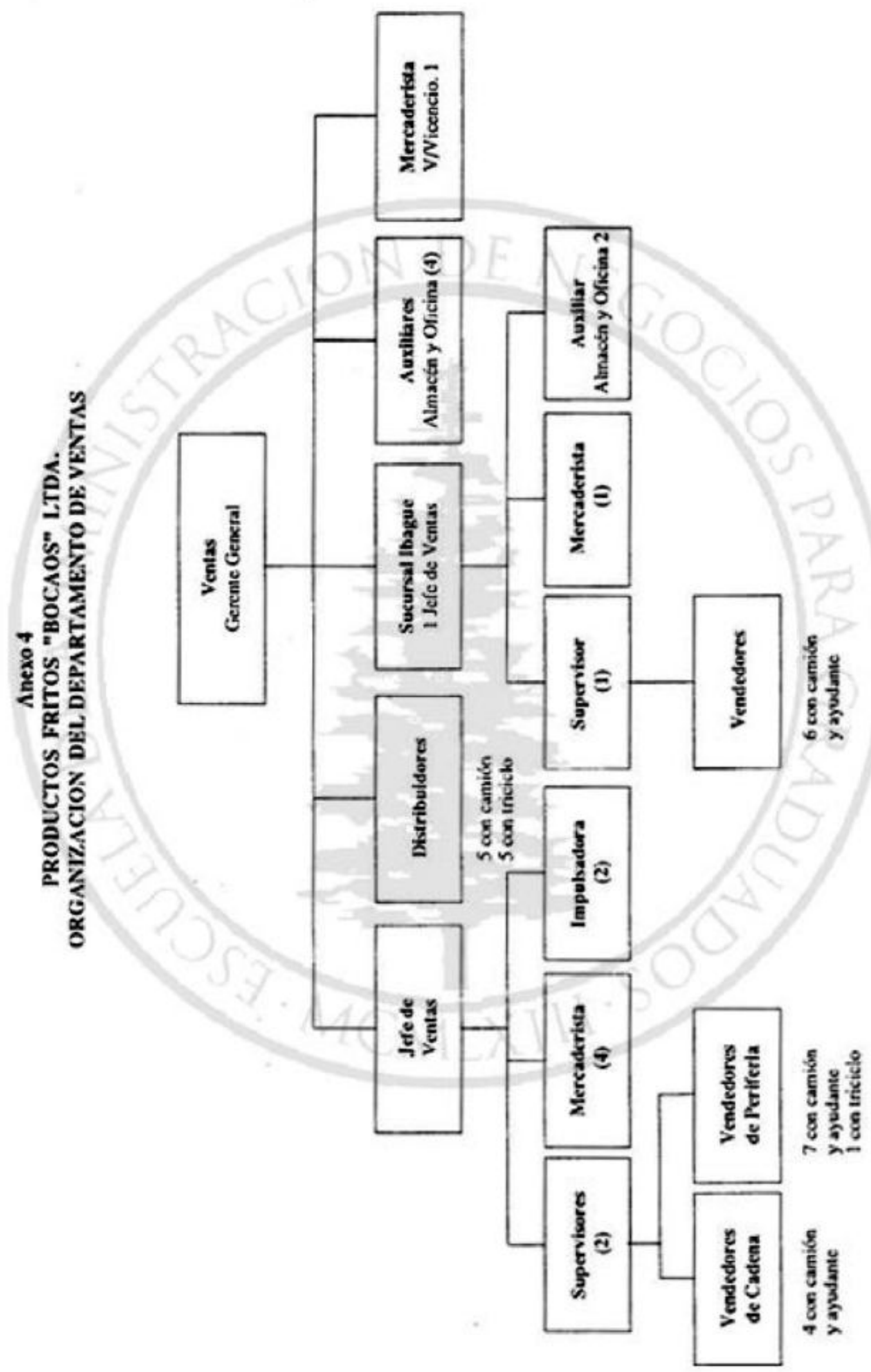

